\title{
Performance Evaluation of the Asymmetric Distributed Lock Management in Cloud Computing
}

\author{
Artur Koçi \\ Epoka University \\ Tirana, Albania
}

\author{
Betim Çiço \\ Epoka University \\ Tirana, Albania
}

\begin{abstract}
Lock management in distributed systems has been the focus for many researchers. The purpose of lock manager is to permit clients to synchronize their activities and agree about their environment. They are techniques used for increasing the availability and reliability of the distributed systems and maintain the consistency of shared files. Based on their behavior and characteristics, lock managers are categorized as centralized lock manger and distributed lock manager. In this paper we present a new distributed lock manager technique that is a fully lock manager. For maintaining file consistency, the lock manager needs to coordinate the tasks with other lock managers in the cloud. This leads to the additional communication that causes extra delays, which can play an important role in defining the quality of the cloud. We are going to analyze in detail these delays and measure the performance of our solution while its implementation is in a different cloud computing environment.
\end{abstract}

\section{Keywords}

Cloud data storage, file consistency, distributed systems, lock manager, voting algorithm, file replication etc.

\section{INTRODUCTION}

Lock management in distributed file systems has been challenging for many researchers. Many developers have been offering different approaches intending to maintain the file consistency in distributed systems. The main purpose of lock managers is to permit clients to synchronize their activities and agree about their environment. The main tasks of the lock management include availability and reliability of the distributed files and maintain the consistency of concurrent access in these files. They are techniques used for synchronizing a set of concurrent requests and prevent the modification of the shared data [1]. When a client delivers a request for accessing a file, a lock manager must first verify if no other request has already been granted permission for accessing it, and only then can grant permission for accessing it and restrict permission for other clients to access that specific file. A lock granted by a client can be shared in two different modes: lock and exclusive lock. Shared lock allows multiple clients to access the same file with the condition that they don't modify it, for example many users can parallelly read the same file. To modify a file, a client must acquire the lock in exclusive mode. Various solutions provide for the election of a leader among a set of servers that will lead and maintain the synchronization between clients and servers and ensure the file consistency. One of the biggest file systems is the Google file system GFS [2]. The architecture of GFS is composed by a single master server and multiple chunkserver [2]. The master server maintains the file system metadata such as namespace, access control and the current location of the file. The client interacts with the master only for metadata operations and the data-bearing communication goes directly to the chunkserver, avoiding the overhead of the master server. To avoid single points of failure, the master server has a replication server. The file concurrency is avoided by creating snapshots and recording appends operation. To keep file consistency, a new lock service called Chubby[3] has been deployed that consists of two main components that communicate via RPC: a cell that is a set of servers also called replicas, and a library that client applications link against. The Client Library maintains all the communications between client applications and servers. The problem of the communication maintaining the distributed consensus has been solved by the Paxos protocol [4] [5]. Replicas use distributed consensus to elect the master that is responsible for reads and writes for the database, while replica copy database from master. After being elected the master periodically sends updates to the replicas to retain the position of master. The model gives a solution to the single of failure but still needs to consume a lot of bandwidth during the communication for maintaining the state replica server. To increase the efficiency of the large scale distributed systems [6] offers a model divided in three levels on the upper level stands for user interface and library that interacts with middle level. The middle one is composed by nodes or servers and every node has three layers global index, local index and data chunks. Each node has local indexes for their own data. Global index keeps a set of shared local indexes by each node and is unique in all nodes. The global index is used for maintaining the consistency for read/write request of the data chunks stored in the cloud. Different applications have been presented different with approaches for resolving the problem of read/write consistency in distributed systems. For example Bayou [7] is a distribution database system that permits separate operations and provides data consistency. Another distributed file system that does not use centralized server is Farsite. The high scalability and availability of Farsite is achieved by using replication [8]. Cassandra [9] is another distributed multidimensional map indexed by the key and implemented by Facebook for maintaining the consistency of their users' data. The core distributed systems technique used in Cassandra are partitioning, replication, failure handling, scaling and membership. Any read/write requests for a key is directed to any of the nodes in Cassandra, then it is the node that determines which replica is responsible for that specific key. Based on their behavior and characteristics, lock managers are categorized as centralized lock manger and distributed lock manager. In centralized lock manager mode exist the possibility that lock manager becomes overload or service becomes unavailable in case of failure of the node acting as lock manger, while in distributed lock manager due to the fact that different nodes execute different files and the lock manger is distributed among different nodes eliminates the drawback of the single point of failure. In his paper [10] proposed the mechanism of scaling lock manger. Each cluster nodes runs an instance of global lock manager and local lock manager. In the local lock manager mode concurrency is locally maintained and in global mode, lock manager cooperates with other cluster nodes to globally synchronize the lock state of the files. There are different techniques for 
maintaining file consistency and increasing the availability and scalability in cloud storage systems [11]. The most wellknown technique is replication [12] which is a technique that replicates each of the files in remote sites. The replica consistency is maintained by different algorithms. Flooding [12] is an algorithm that propagates the update of the replicas any time that one of the replicas has been updated. The replication technique is a sustainable method that increases the availability and scalability of cloud storages but consumes a lot of space for storing the replicas and uses a lot of redundant messages which increase the communication overhead. A large amount of research has been carried out in this field for deploying a new technique to reduce the storage consumption. To reduce the number of replicated nodes, one technique for regenerating lost nodes is to use existing nodes. In such systems, each file is divided into an equal size of bytes per parts and stored randomly in nodes by a technique so that each of the nodes will be reassembled by using a subdivision of the total nodes [13]. The maximum distance separable (MDS) erasure codes described in [13], [14] are erasure codes techniques that can generate a node by contacting a subset of $k$ nodes instead of $n$ nodes where $n$ is the total number of parts and $\mathrm{k}$ is smaller than $\mathrm{n}$. Compared to replication, the main disadvantage of the MDS codes is the high bandwidth consumption. In this paper we are going to analyze the performance of the lock manger algorithm offered by [15]. The core structure of the algorithm is based on the concept presented by [1]. In contrast to his approach, which is a centralized solution, this algorithm provides a fully distributed solution with no election of a master. Every server acts as a master for the received requests and communicates with other servers only when it is necessary. The author adds the concept of the client request migration. When concurrent access in files occurs, most of lock algorithms are designated in a way that one node waits for the other node to finish and then grants permission for accessing the file while in this approach the request is migrated from initiator node to the end node that has already granted access to the mentioned file. Using this approach, we decrease the time that the request must wait in the initiator node queue. After the request has been migrated, the initiator server is free to accept a new request. Our contribution in this paper is to analyze the performance of this algorithm when it is integrated with different cloud platforms. According to their usage, cloud platforms are categorized in two main categories: big size cloud, such public clouds, and small cloud size. The performance of the lock manager can be affected by the amount of cloud usage. The Asymmetric Distributed Lock Management in Cloud Computing is designed to execute incoming request in three different modes:

\section{a. Self-management mode}

\section{b. Owner server management mode}

\section{c. Remote server lock management mode.}

In Self-management mode the server is the imitator, owner and the executer, no extra communication among other servers is added. In the other two modes, to keep the consistency of the shared file, extra communication is added. We have named it Time until Granted. Things in the cloud happen out of normal control and it is obvious that the three modes will occur many times. This is the main factor that will affect the performance of our algorithm.

In order to get good samples and analyze the sustainability of this algorithm, we define the number of client requests delivered in a moment of time as a benchmark, in random mode, with fix configuration resources of the cloud. We repeat the same test two more times, keeping the same benchmark and changing the cloud configuration resources. The same tests are performed in two more phases by using the same resource configuration as in the first phase with different benchmarks. The results achieved are discussed in detail in section 4 .

\section{ADLMCC - ASYMMETRIC DISTRIBUTED LOCK MANAGEMENT IN CLOUD COMPUTING}

The ADLMCC functionalities are described in detail in [15]. In this section we will explain the main concepts that ADLMCC provides and the method used to prevent concurrent access in files and maintaining their consistency. File concurrency can occur with requests delivered to the same server and between requests delivered to different servers. A lock manager of one server collaborates with lock managers of the other server to control concurrent access to files. This process is carried out by using inter-process communication among lock managers components. The lock manager runs as an individual process on every server and cooperates with other lock managers only when needed for maintaining the inconsistency of the files.

The data structures of the lock manager are composed of six important key factors that maintain file consistency when concurrent access happens. The six key factors are Server Node Table (SNT). File Directory (FD), Requesting Lock Table (RLT), Migrate-out Table (Mout-T), Migrate-in Table (Min-T), Locked File List (LFL). Each lock manager has two different sets of data structure. One that is maintained locally and that is not shared with any of the other servers and a second data structure that is fully distributed among the other servers. SNT is responsible for memorizing the configurations of all servers in the cloud. The information memorized from SNT is the server's ID that is unique and is used for recognizing the server among other server parts of the same cloud, The Server status and the switchover server are used in case of main server failures. SNT is fully synchronized among all other servers. FD is the directory that determines the path of each file stored in nodes and that is the server that creates the file and has the ownership rights for a specific file. We agree that FD is a data structure with pairs of file names and server numbers and is fully synchronized in the cloud. Min-T and Mout- $T$ respectively maintain information about achieved permission for accessing specific file from another server and the list of files that their lock has been migrated to other servers. RLT stores the list of all locks a server has requested. The information kept in RLT consists in user requester ID, the file name requested, the lock mode and timestamp. The table is maintained locally and works for managing locks locally in the server. LFL contains all the necessary information for locks that a specific lock manager is managing and responsible for. The set of attributes LFL contains are sets of: requesters ID, requester server, file name, lock mode and a timestamp; and a further set of two lists for queuing lock requests: one for granted and the other for blocked locks. According to the lock state of a specific file, the lock manager locally maintains the consistency in the files and only in case of necessity cooperates with the others for the lock acquitting to perform lock request operations. As a generic solution, a lock management algorithm has to follow the sequence of one to four steps to archive the lock to a file and responds to the 
client as described in Fig. 2. The sequence from one to four procedures is as following:

- $\quad$ Self-management of shared locks in servers

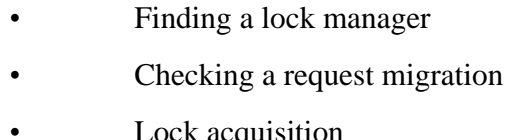

- $\quad$ Lock acquisition

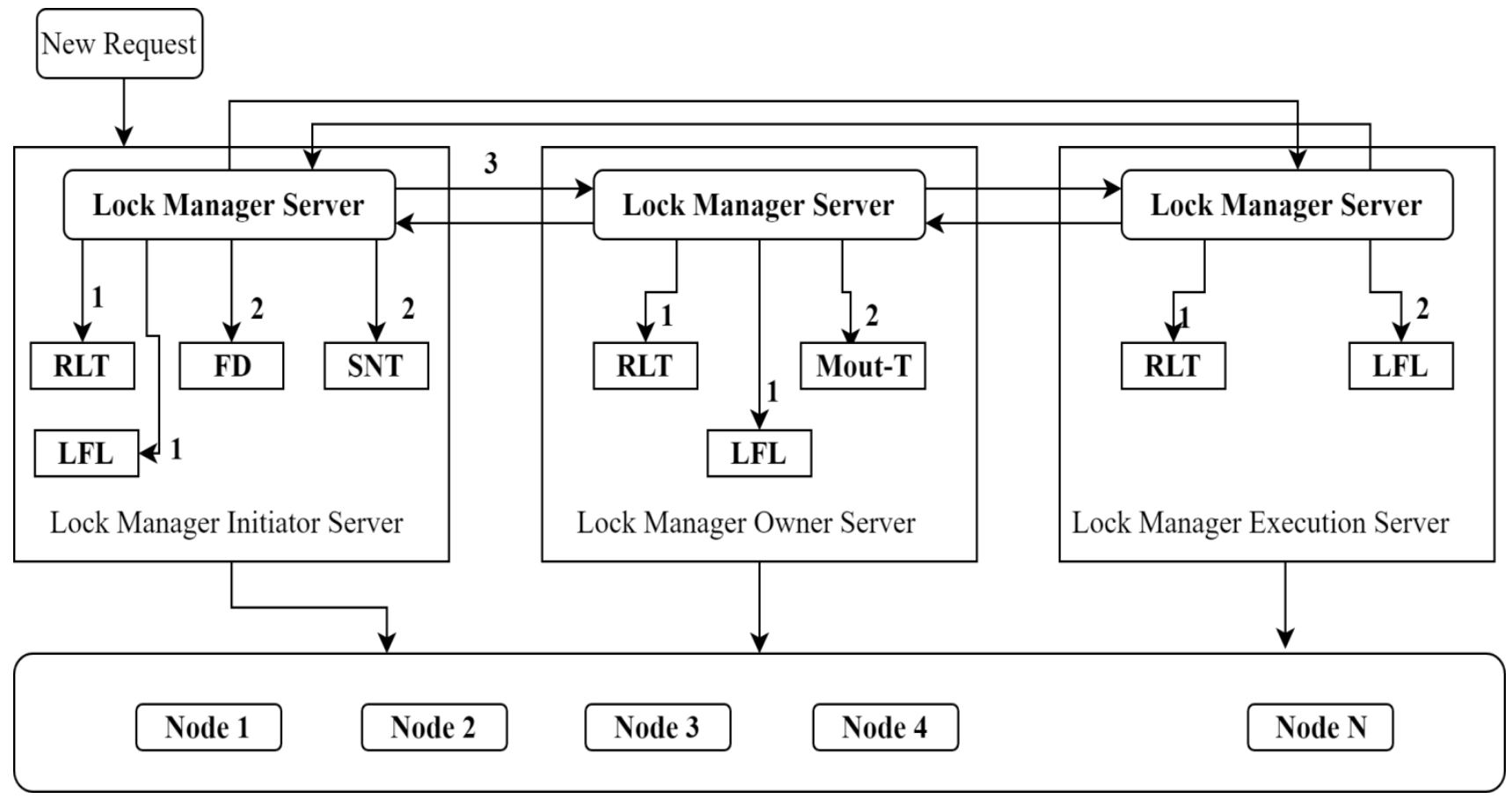

Fig 1: ADLMCC Architecture

\subsection{Self-Management Of Shared Locks In Servers}

The end users issue a request to one of the servers to access specific files in the storage nodes. The server lock manager, after receiving the request, controls if the file name requested is already in the table of its RLT. If the file name does the lock manager inserts the request in the table of RLT associating the requester ID and its related operations for the requested file. The next step is to add the request to the LFL table and to further procedures. After receiving the request, the LFL starts checking the lock state already applied to the file for acquitting the right to read or write the file in the request. In case the file is not found in RLT, the lock manager does not yet have the necessary information for the state of the file and checks in FD to know the owner of that file and in case that it is owner, controls in Mout-T if permission has already been given to execute the file.

After ensuring that the file is not found in Mout-T, the lock manager has sufficient information for the lock status on the file and adds the file for execution, otherwise the file is found in Mout-T. The lock manager, after getting the servers that are executing the file from Mout-T, transfers the file for execution to that server and removes the file from RTL. Considering this scenario, when the initiator server is the owner for that specific file and the request either is found in RLT or is not inserted in Mout-T, the request is set to the LFL table for execution. LFL receives the request and communicates with nodes to know the availability of the chunk stored among them. Each request issued from the server to the nodes has a unique request ID. Nodes will be available if there is no lock on them, and if there is a lock, then the availability will depend on the type of lock. If they are available for a certain request, they will supply to the server the adequate information. The information that they will provide consists of their lock status, node number and request id. The server collects the information and checks in the lock table if that node can be granted to that lock. If it can be granted, it updates its lock table with the corresponding lock as the end user request id, and then updates the lock bit on the node. It would not be necessary to update the lock bit if the node on which the request is is the same as the recent lock one because these requests will be read-read, read-repair or repair-repair and all these have same vote bit. The request is managed locally by the lock manager. Processing the lock request is completed without interacting with other lock managers on other servers. The relationship diagram is explained in Fig. 4.

In case the file name does not exist in RLT and the server is not the owner, it is necessary to first acquire the execution right for the file by cooperating with lock manger owner and the other lock managers as described next.

\subsection{Finding A Lock Manager}

The first procedure is to look up the FD and find the lock manager server responsible for the file. Next, the lock manager checks on its SNT to discover the owner server and ensure it is alive and still in the cloud. 


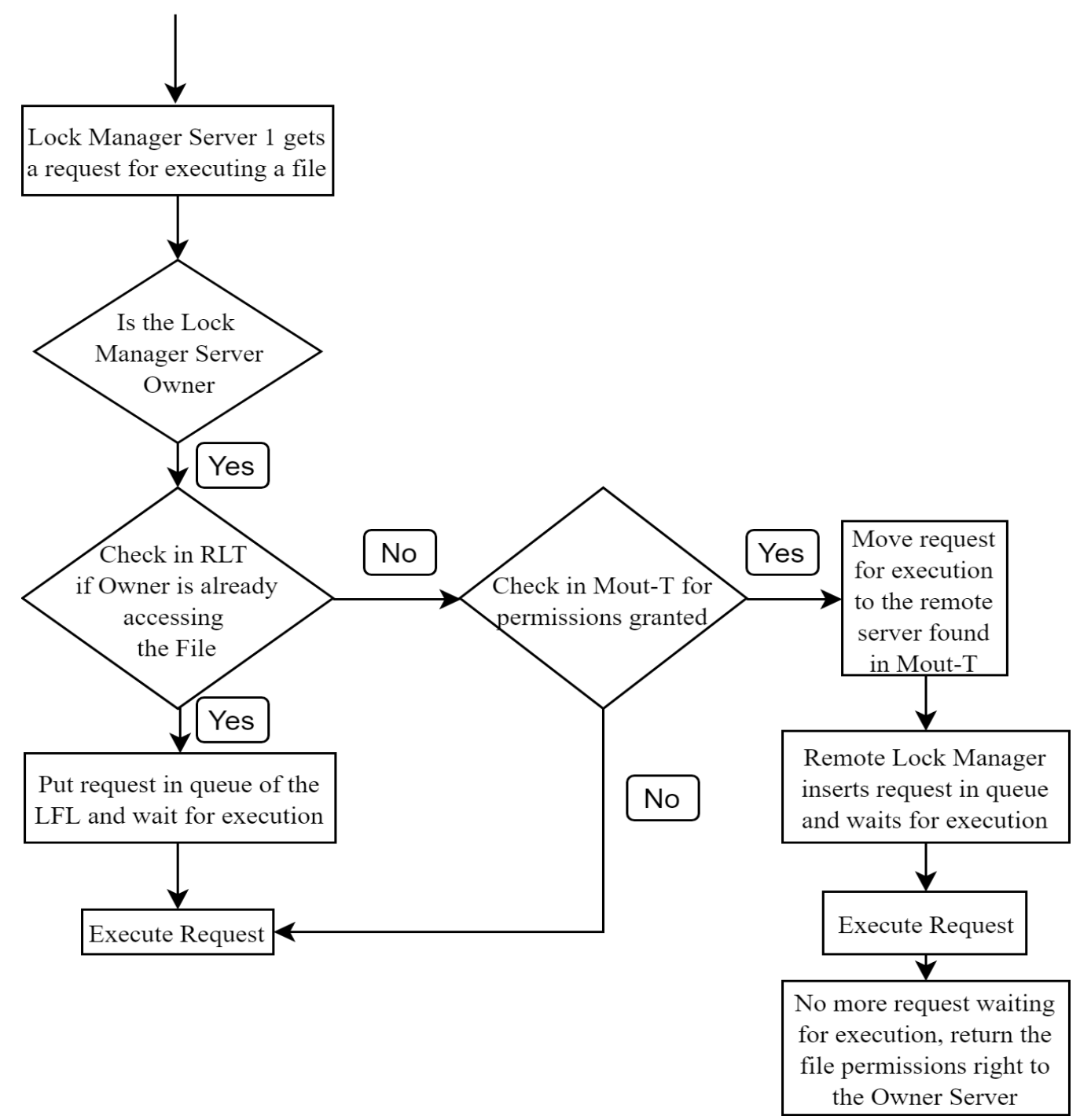

Fig 2: Self-management of the File Permissions

If the Server is alive in the Cloud, the lock manager on that server will be taken as responsible for the file. If not, the switchover Server in the table of SNT is engaged as the alternative node for the lock management. The lock manager at the lock initiator server sends a lock request message to the lock manager on the node found in the above procedures.

\subsection{Checking A Request Migration}

The lock manager that receives a lock request message from the lock request initiator server is responsible for managing the lock on the file. The manager checks in the table of RLT and on Mout-T if any lock is applied to the file or the lock management is migrated to a lock manager on another server. In case the name of the file is not found in RLT, or in Mout-T, the responsible server places the file in Mout-T and the lock management is migrated to the server initiator. The server initiator grants lock management for the file, as described in Fig. 3 In case the file name on the request is found in the RLT of the owner server, then that means the server has locked the file. The responsible server informs the server initiator and the request is migrated from the server initiator to the owner server and is preceded from there. This relationship is explained in Fig. 4. In case that the name of the file in the request is found on Mou-T, the owner servers replies to the initiator with the ID of the server that has granted access to that file, and the initiator server with the information taken from the owner, migrates the request to that server, and the request is progressed from that specific server. In doing so, it is the remote server that will now reply to the request and the server initiator removes the request from its queue and it proceeds to another request. The initiator server does not keep any track of that request and does not get any feedback regarding whether there is an execution. In Fig.5. we have the relationship diagram that graphically explains that specific situation.

\subsection{Lock Acquisition}

The lock manager receives the request and updates its RTL, updates the LFL Table with the filename and with its attributes, and informs the server initiator that the request has been added to the list for execution. Now the remote server is the one that will reply to the request and the server initiator removes the request from its queue and proceeds to another request. The initiator server does not keep any track of that request and does not get any feedback regarding the execution. The initiator server removes the request from its RLT table. After migration of the request from the server initiator, the server starts sending votes to nodes for $\mathrm{r} / \mathrm{w} / \mathrm{rep}$ the file in the request. 


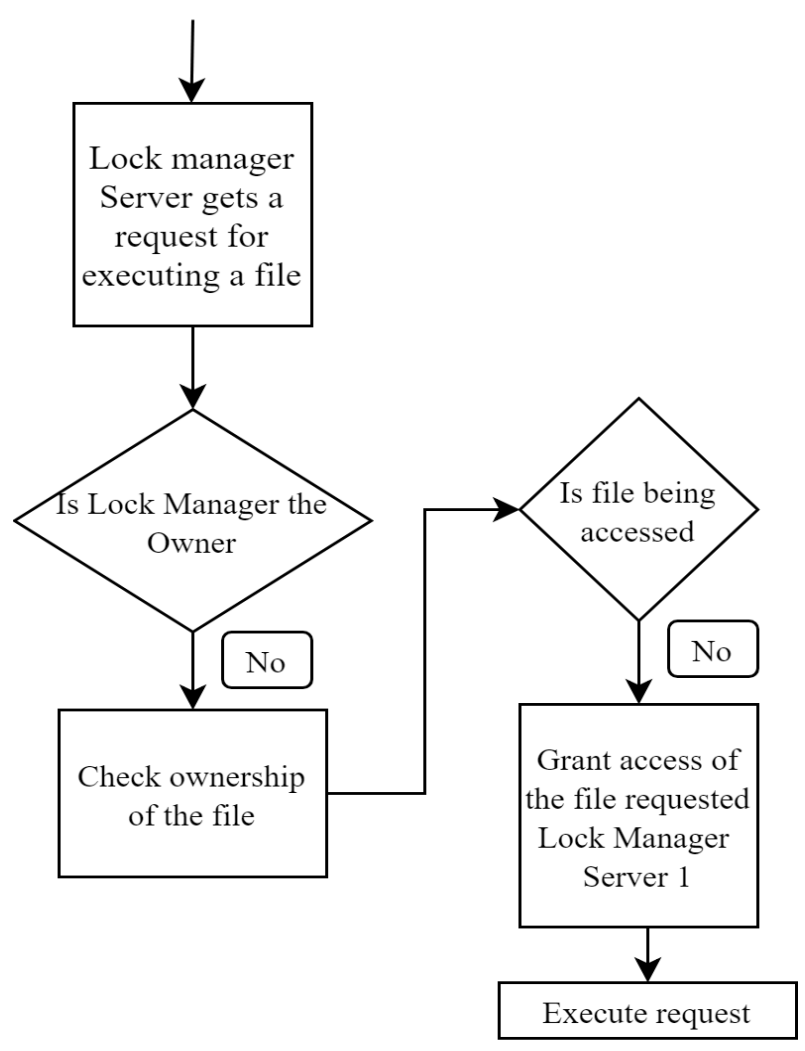

Fig 3: Initiator Server gets File Execution Permissions from Owner Server

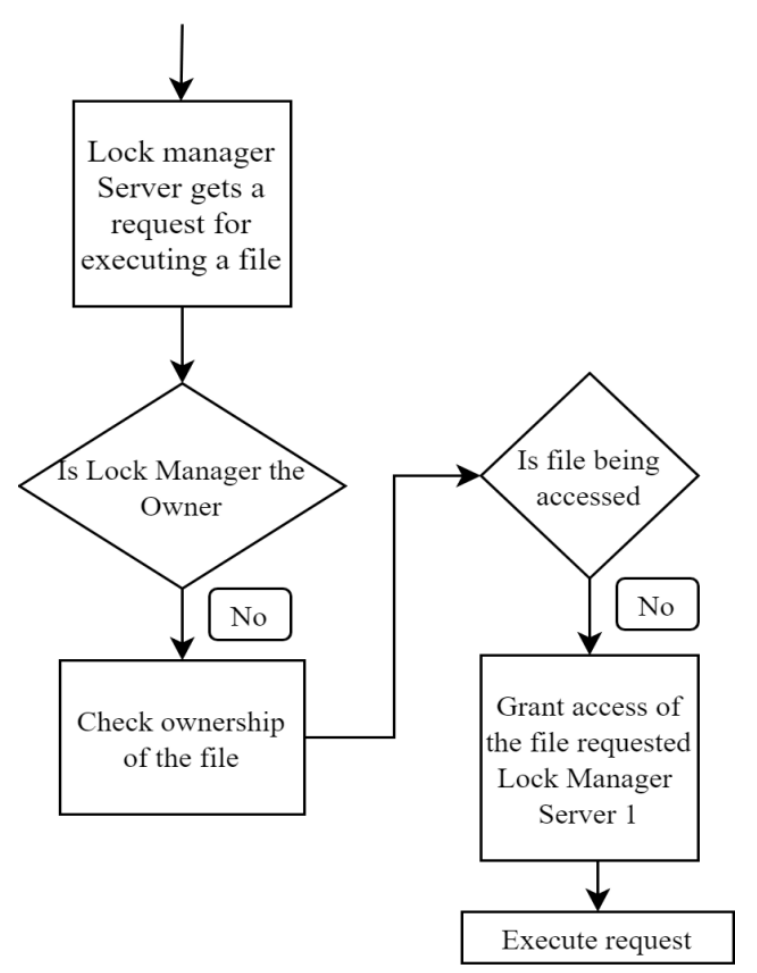

Fig 4: Request Execution Migration from Initiator Server to Owner Server

Now the remote server is the one that will reply to the request and the server initiator removes the request from its queue and proceeds to another request. The initiator server does not keep any track of that request and does not get any feedback regarding the execution. The initiator server removes the request from its RLT table. After migration of the request from the server initiator, the server starts sending votes to nodes for $\mathrm{r} / \mathrm{w} / \mathrm{rep}$ the file in the request.

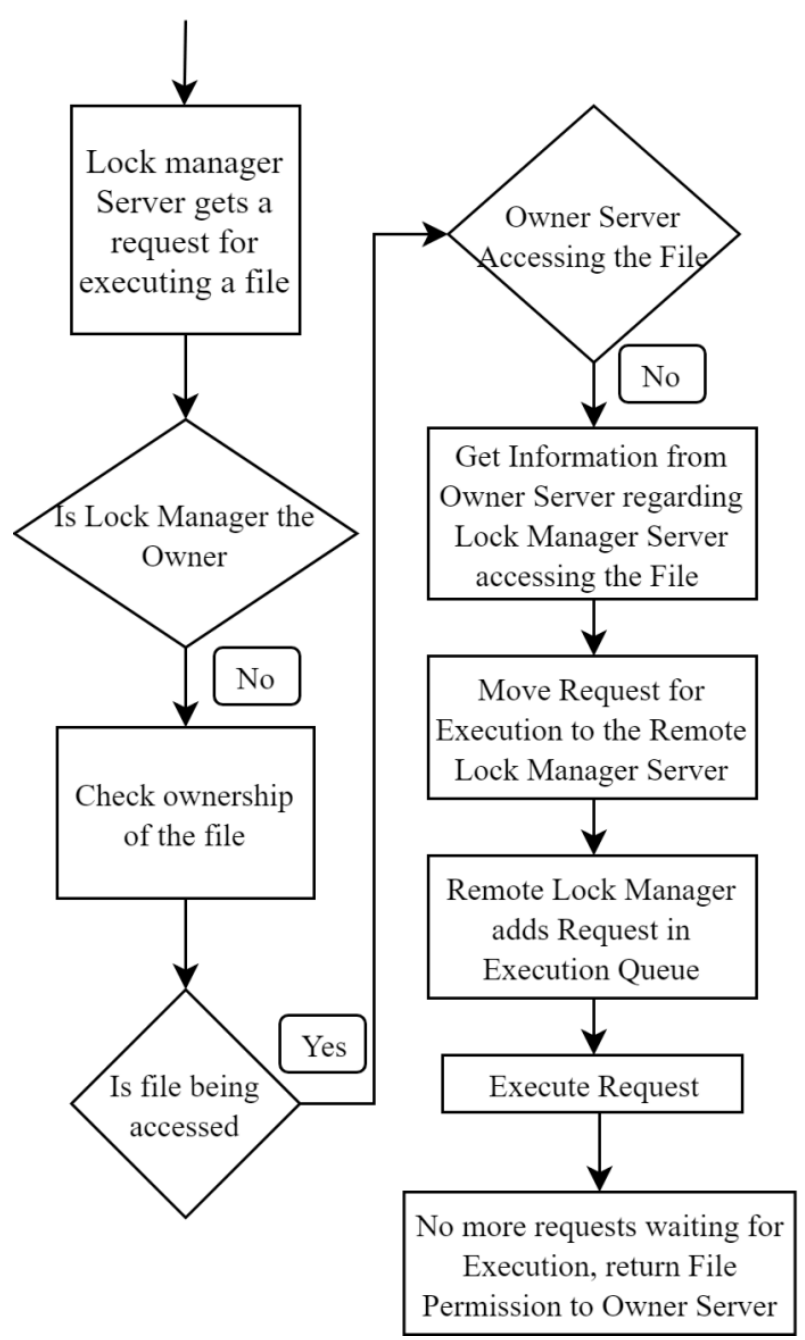

Fig 5: Request Execution Migration from Initiator Server to Remote Server

\section{EXPERIMENTAL RESULTS AND DISCUSSION}

In section 2 we explained the main concepts that ADLMCC uses to maintain the consistency in distributed systems that are realized in three different approaches that consist of a. Self-management of shared locks in server, $b$. owner server management, c. remote server lock managements. According to [2], once written, a file is read-only, and it is certain that there is a high probability that read/write requests of the clients for a specific file are delivered to the server that created the file. This approach implies a sustainable solution and avoids all other communication with other servers in the cloud.

Nevertheless, there will be many other requests delivered to different servers requesting the attribution rights of a certain file. This new condition leads to another state where the server receiving the request has to collaborate with other servers to maintain file consistency. For the simplicity of our work we call this imitator server. The amount of communication between servers plays the biggest role in the system performance adding a delay that will differ based on 
the communication media and the number of the request in a certain time. Many cloud solutions are developed according to the type of the service and it is essential for the developers to find an equilibrium between resources and purpose of usage. In this paper we measured the sustainability, delay and performance of the lock manager algorithm and analyzed its behavior while implementing it in big data clouds, mid-size and small size clouds. According to the architecture of the lock manager, the delay added in self-management is zero, there is no communication with other servers and the execution time depends only on the number of the request that the server itself receives. The biggest concern is the additional delay which occurred during the communication to find the state of file and to decide which servers are responsible for executing that request. We refer to this delay as Time until Granted and was the focus of our analysis. In the first test we defined the number of requests as a benchmark and we kept it unchanged throughout the process, alternating the number of servers, nodes and files stored on these nodes. Throughout our discussion, the requests were delivered asymmetrically to the servers and were delivered at the same time and randomly.

Fig. 6. shows the Time until Granted for 100 requests delivered in random mode and the cloud is composed by 5 servers, 3 nodes, 30 files and 5 clients.

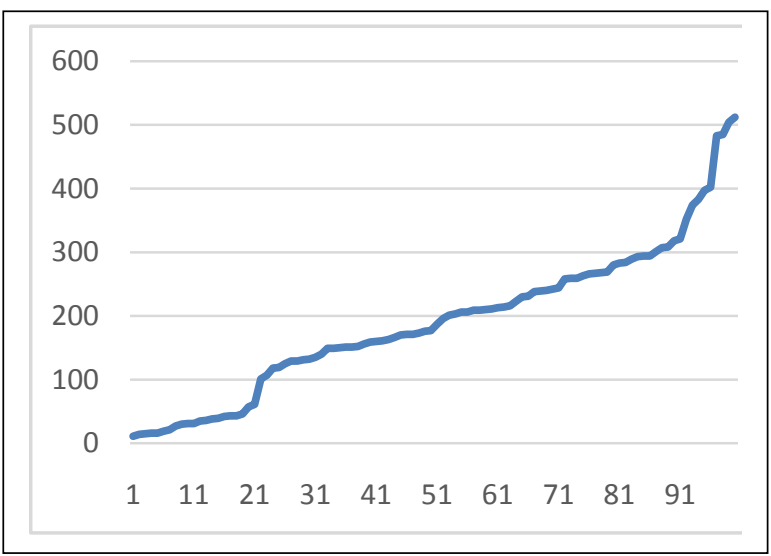

Fig 6: Time until Granted for 100 requests in random with 5 servers

Looking at the cloud resources composition, it's clear that this is an example of a small sized cloud which is composed by a few servers and nodes. Bearing in mind the number of requests, every client executes more than one request and a file is requested from more than one client with a different operation mode. Because of the small number of requests, we realize that the Time until Grated is very insignificant. The times differ from near zero and the highest delay is 600 milliseconds. For most of the requests Time until Grated is less than 300 milliseconds.

As the number of requests increases, we note that the graph encompasses a nonlinear change of the Time until Grated. Following the same explanation, in Fig. 7 we illustrate the example with 100 requests delivered randomly and the cloud composition now is made of 10 servers, 10 nodes, 300 files and 50 clients. With the growth of the number of servers and files, the probability for requests to contact the same file is reduced. Due to that, we can observe from the graph that the overall Time until Grated for executing 100 requests is reduced by half. In line with the first example, with the increase of the number of requests, the time that a request has to wait starts growing. In contrast to the first example, the increase is much more linear and now the total Time until Grated is only 300 milliseconds

We continued to increase the number of resources, for the same number of requests, and now the number of servers is 30 and number of other resources is 20 nodes, 1000 files and 200 clients. With the new changes, the cloud resources extend so that the number of clients and the files stored in nodes is much bigger than the total number of requests. The probability that a client delivers more than one request and that two requests access the same file is almost zero.

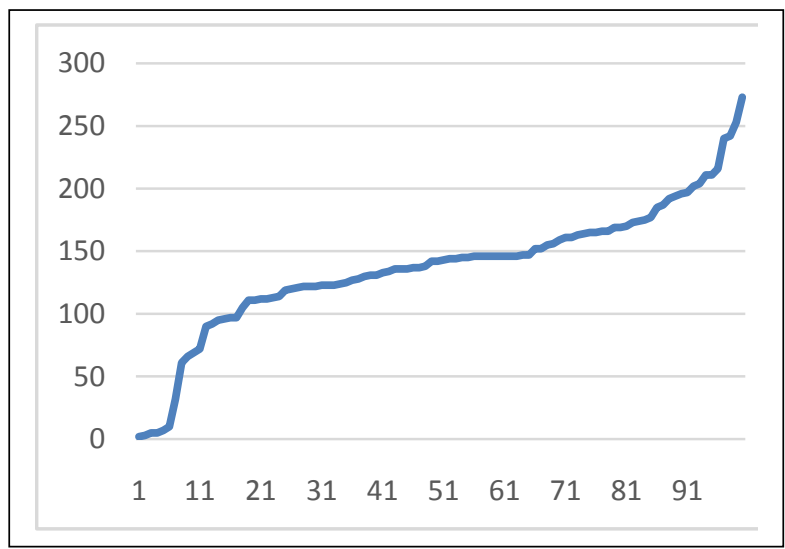

Fig 7: Time until Granted for 100 requests in random with 10 servers

The results received, as illustrated in Fig. 8. indicate that the overall Time until Grated continues to decrease, and the graph curve is almost linear. The Time until Grated is less than 250 milliseconds and there is no moment in which the time rises very quickly; everything is smooth with no specific moment to be investigated.

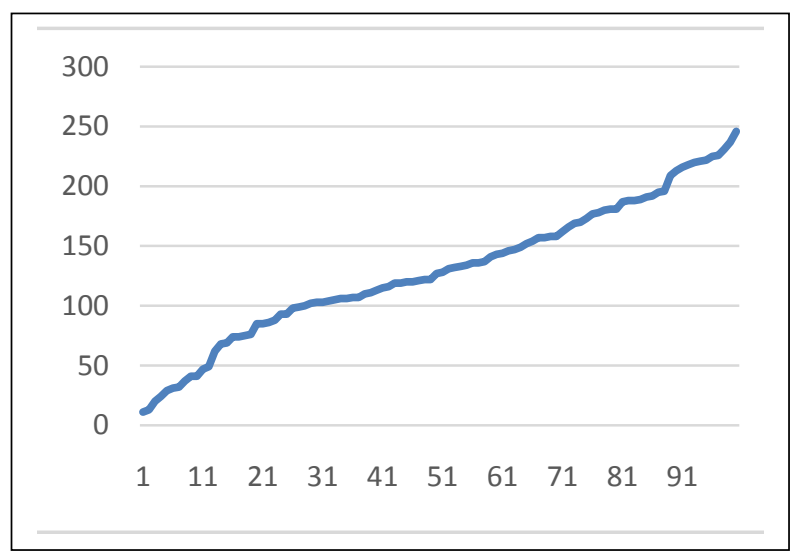

Fig 8: Time until Granted for 100 requests in random with 30 servers

In comparison with the first implementation, to better understand how Time until Granted defers according to the cloud resources and its usage, we will have held the same study only by modifying the parameter of our benchmark. Now the number of the requests has changed from 100 to 500 requests. The cloud is composed of 5 servers, 3 nodes and 30 files. All the requests for file are delivered by a mere 5 clients. As per the first analysis, which took into account the cloud composition, there was a high probability that a high number of requests were delivered by the same client and a high probability that different requests ask to read/write the same file. 
In Fig. 9. we have given the variance of Time until Granted in milliseconds for 500 requests. It is clearly depicted that with the growth of the total number of requests, the overall Time until Granted is increased. The graph is almost linear for most of time and after a certain request it changes exponentially. It is notable from the graph that until 450 requests the time increases linearly and the maximum time for a request to wait is nearly 650 milliseconds, after this point time it goes up very quickly and at request number 500 the Time until Grated goes to 1400 milliseconds.

At this point, from a change of 50 requests we denote a change of time that varies from 650 to 1400 milliseconds. As for the first corresponding tests, the curves changes are almost similar to each other's. Another aspect that will affect the curve linearity is the type of request delivered as the more write requests delivered first the more Time until granted is needed.

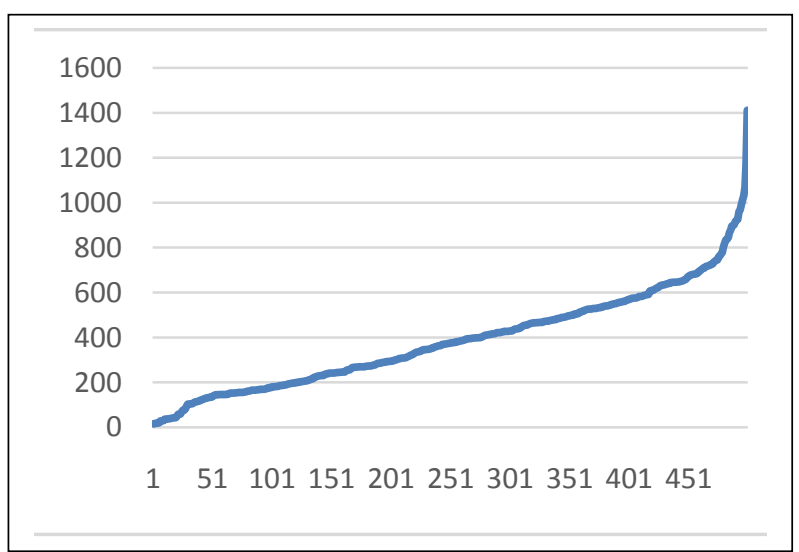

Fig 9: Time until Granted for 500 requests in random with 5 servers

One remarks to be mentioned in Fig. 9. is that for the given composition of cloud resources, after a certain number of requests the time increases very rapidly. Following the same explanation, we keep to 500 the number of requests and we change the number of servers to 10 , and the number of other resources becomes 10 nodes, 300 files and 50 clients. Still, there will be a high probability that every client delivers more than one request and a high probability that many requests ask to $\mathrm{read} / \mathrm{write}$ the same file.

The graph on the Fig.10 illustrates how the Time until Granted changes for the execution of 500 requests in the new settings.

As notable from the graphs above, there is a similarity between Fig. 9 and10. The increase of the resources did not provide any improvement in the overall performance. After a certain number of requests, the linearity of the graph breaks and the Time until Grated raises exponentially. For the same number of requests, we increased the number of servers to 30 and the number of other the resources becomes respectively 20 nodes, 1000 files and 200 clients. Within the new configuration, the probability that a client delivers more than one request and that the same server receives more than one request from clients remains significant. Considering the number of files, the probability that the same file is requested from more than one request is decreased. The new results are described in Fig 11. which explains how the Time until Granted varies for executing 500 requests with 30 servers.

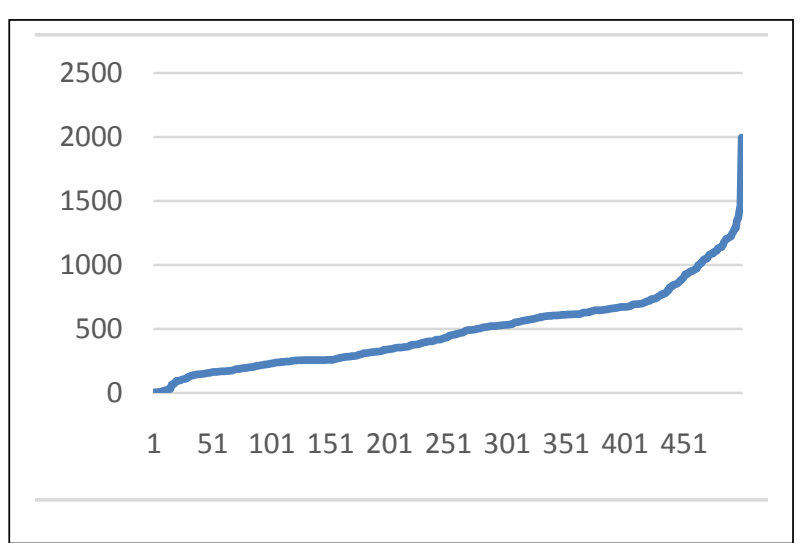

Fig 10: Time until Granted for 500 requests in random with 10 servers

With the increase of the resources, even though there still is a high possibility for each client to deliver more than one request and for each server to execute more than one request, we can denote that the overall Time until Granted has decreased. The linearity remains almost unchanged for the entire process. We can note that in this new state it has reached equilibrium between the amount of resources and the number of the requests.

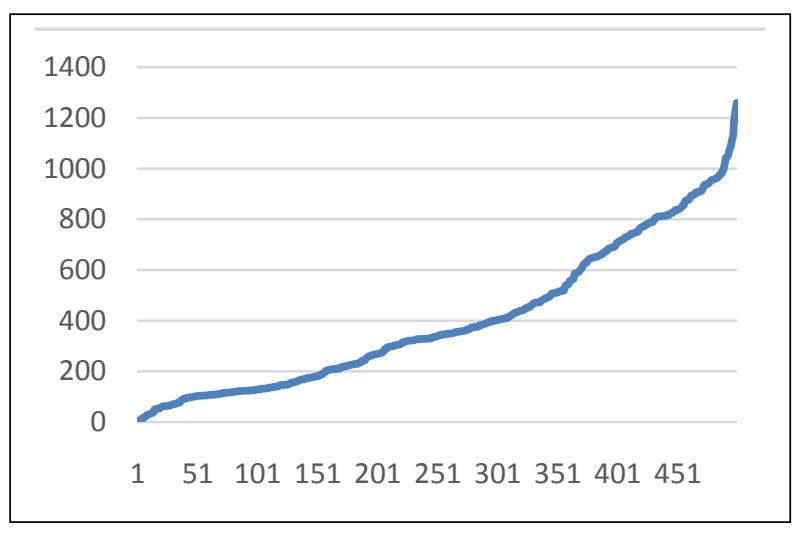

Fig 11: Time until Granted for 500 requests in random with 30 servers

If more resources are added, the more Time until Granted will decrease and if more number of requests are delivered, more time is required for the overall execution. This is a parameter that requires the attention of developers and needs to be optimized for having the desired performance of the cloud.

\section{CONCLUSION}

In this paper we have analyzed the performance of ADLMCC when it is implemented in different cloud environments. The lock manager is the solution for the implementation of different cloud storages.

According to its characteristics, ADLMCC is a fully distributed solution and eliminates the need for a master node which is going to control and maintain the consistency of shared files. Our approach implements most of the key factors for a cloud to be a reliable and fault tolerant cloud. On the other hand, it keeps the file consistency with the least possible communication among other colleagues. Based on this design, communication with other lock managers happens only when extra information is needed

One of the factors that define the quality of the service for our solution is the time to wait for a client until its request is 
processed. This time is determined from the communication between lock managers for the consistency of the file that has been named as Time until Granted, and from the availability of the requested files. Time until Granted was the focus of this paper and was analyzed in detail.

According to our test results, we can conclude that Time until Granted is low for a normal load implemented in small and mid-size clouds and becomes significant when the number of requests is increased.

Another conclusion achieved from our analysis is that, with the increase in resources, there is also an increase in the performance of the cloud and the Time until Granted decreases significantly. The Time until Granted depends more on the type of the request. For any increase for the delivered write requests more time is required for the execution process, and for read request less is required.

The lock manager algorithm offered is a sustainable solution and fits better in cloud storages that require precision on the data modification.

\section{REFERENCES}

[1] Kishida, H., and Yamazaki, H.: 'SSDLM: architecture of a distributed lock manager with high degree of locality for clustered file systems', in Editor (Ed.)^(Eds.): 'Book SSDLM: architecture of a distributed lock manager with high degree of locality for clustered file systems' (IEEE, 2003, edn.), pp. 9-12

[2] Ghemawat, S., Gobioff, H., and Leung, S.-T.: 'The Google file system', SIGOPS Oper. Syst. Rev., 2003, 37, (5), pp. 29-43

[3] Burrows, M.: 'The Chubby lock service for looselycoupled distributed systems', in Editor (Ed.)^(Eds.): 'Book The Chubby lock service for loosely-coupled distributed systems' (USENIX Association, 2006, edn.), pp. 335-350

[4] Lamport, L.: 'The part-time parliament', ACM Transactions on Computer Systems (TOCS), 1998, 16, (2), pp. 133-169

[5] Lamport, L.: 'Paxos made simple', ACM Sigact News, 2001, 32, (4), pp. 18-25

[6] Wu, S., and Wu, K.-L.: 'An Indexing Framework for Efficient Retrieval on the Cloud', IEEE Data Eng. Bull., 2009, 32, (1), pp. 75-82
[7] Terry, D.B., Theimer, M.M., Petersen, K., Demers, A.J., Spreitzer, M.J., and Hauser, C.H.: 'Managing update conflicts in Bayou, a weakly connected replicated storage system' (ACM, 1995. 1995)

[8] Adya, A., Bolosky, W.J., Castro, M., Cermak, G., Chaiken, R., Douceur, J.R., Howell, J., Lorch, J.R., Theimer, M., and Wattenhofer, R.P.: 'FARSITE: Federated, available, and reliable storage for an incompletely trusted environment', ACM SIGOPS Operating Systems Review, 2002, 36, (SI), pp. 1-14

[9] Lakshman, A., and Malik, P.: 'Cassandra: a decentralized structured storage system', ACM SIGOPS Operating Systems Review, 2010, 44, (2), pp. 35-40

[10] Choi, S., Choi, M., Lee, C., and Youn, H.Y.: 'Distributed lock manager for distributed file system in shared-disk environment', in Editor (Ed.) $)^{\wedge}$ Eds.): 'Book Distributed lock manager for distributed file system in shared-disk environment' (IEEE, 2010, edn.), pp. 2706-2713

[11] Buyya, R., Yeo, C.S., Venugopal, S., Broberg, J., and Brandic, I.: 'Cloud computing and emerging IT platforms: Vision, hype, and reality for delivering computing as the 5th utility', Future Generation computer systems, 2009, 25, (6), pp. 599-616

[12] Tewari, S., and Kleinrock, L.: 'Analysis of search and replication in unstructured peer-to-peer networks', in Editor $(\text { Ed. })^{\wedge}($ Eds.): 'Book Analysis of search and replication in unstructured peer-to-peer networks' (ACM, 2005, edn.), pp. 404-405

[13] Dimakis, A.G., Godfrey, P.B., Wu, Y., Wainwright, M.J., and Ramchandran, K.: 'Network coding for distributed storage systems', IEEE transactions on information theory, 2010, 56, (9), pp. 4539-4551

[14] Dimakis, A.G., Ramchandran, K., Wu, Y., and Suh, C.: 'A survey on network codes for distributed storage', Proceedings of the IEEE, 2011, 99, (3), pp. 476-489

[15] Koçi, A., and Çiço, B.: 'ADLMCC-Asymmetric distributed lock management in cloud computing', Unpublished paper, 2018 